\title{
Correction of glycogen storage disease type III with rapamycin in a canine model
}

\author{
Haiqing Yi • Elizabeth D. Brooks • Beth L. Thurberg • \\ John C. Fyfe • Priya S. Kishnani • Baodong Sun
}

Received: 16 August 2013 /Revised: 27 December 2013 / Accepted: 23 January 2014 / Published online: 8 February 2014

(C) Springer-Verlag Berlin Heidelberg 2014

\begin{abstract}
Recently, we reported that progression of liver fibrosis and skeletal myopathy caused by extensive accumulation of cytoplasmic glycogen at advanced age is the major feature of a canine model of glycogen storage disease (GSD) IIIa. Here, we aim to investigate whether rapamycin, a specific inhibitor of mTOR, is an effective therapy for GSD III. Our data show that rapamycin significantly reduced glycogen content in primary muscle cells from human patients with GSD IIIa by suppressing the expression of glycogen synthase and glucose transporter 1 . To test the treatment efficacy in vivo, rapamycin was daily administered to GSD IIIa dogs starting from age 2 (early-treatment group) or 8 months (late-treatment group), and liver and skeletal muscle biopsies were performed at age 12 and 16 months. In both treatment groups, muscle glycogen accumulation was not affected at age 12 months but significantly inhibited at 16 months. Liver glycogen content was reduced in the early-treatment group but not in the latetreatment group at age 12 months. Both treatments effectively reduced liver fibrosis at age 16 months, consistent with markedly inhibited transition of hepatic stellate cells into myofibroblasts, the central event in the process of liver fibrosis. Our results suggest a potential useful therapy for GSD III.
\end{abstract}

H. Yi • E. D. Brooks • P. S. Kishnani • B. Sun $(\bowtie)$

Department of Pediatrics, Duke University Medical Center, Durham, NC 27710, USA

e-mail: baodong.sun@duke.edu

\section{B. L. Thurberg}

Department of Pathology, Genzyme, a Sanofi Company, Framingham, MA 01701, USA

J. C. Fyfe

Laboratory of Comparative Medical Genetics,

Michigan State University, East Lansing, MI 48824, USA

\section{Key messages}

- Rapamycin inhibited glycogen accumulation in GSD IIIa patient muscle cells.

- Rapamycin reduced muscle glycogen content in GSD IIIa dogs at advanced age.

- Rapamycin effectively prevented progression of liver fibrosis in GSD IIIa dogs.

- Our results suggest rapamycin as potential useful therapy for patients with GSD III.

Keywords Rapamycin - Glycogen storage disease type III Glycogen accumulation $\cdot$ Hepatic fibrosis

\section{Introduction}

Glycogen storage disease type III (GSD III) is an autosomal recessive disorder caused by deficiency of the glycogen debranching enzyme and accumulation of structurally abnormal glycogen in multiple tissues. Most patients have involvement in both liver and muscle (type IIIa), but in $\sim 15 \%$ of patients, only liver is affected (type IIIb) $[1,2]$. There is significant variability of clinical signs referable to liver, skeletal muscle, and myocardial dysfunction. Liver symptoms typically appear in childhood and lessen with age; liver cirrhosis, hepatic adenomas, and hepatocellular carcinoma occur in some cases [1, 3-6]. Progressive myopathy and/or cardiomyopathy is a major cause of morbidity in adults. Muscle weakness present during childhood often becomes more prominent in adults $[1,7,8]$. Ventricular hypertrophy is a frequent finding in GSD IIIa, and sudden death caused by cardiac arrhythmias or cardiac failure has been reported [9-12]. Current treatment is symptomatic, and there is no effective therapy for the disease [1]. Recently, we have reported on the natural history and disease progression in a canine model of GSD IIIa [13]. We demonstrated that the clinical 
course of this model closely resembles that of human disease. Affected dogs develop progressive hepatic fibrosis and muscle damage with increasing age [13].

The mammalian target of rapamycin (mTOR) plays an important role in regulating cell growth and proliferation, protein synthesis, and metabolism in response to nutrients, growth factors, and stress through a variety of signaling pathways [14-16]. Dysregulation of mTOR has been implicated in several human diseases including cancer, diabetes, obesity, cardiovascular diseases, neurological disorders, and some age-related diseases. Inhibition of mTOR function has shown great potential as a novel therapy for these diseases [17-19]. Rapamycin, a specific inhibitor of mTOR, is an FDAapproved immunosuppressive drug to prevent rejection in organ transplantation. Pre-clinical studies showed that rapamycin affects glycogen and glucose metabolism. In vitro, rapamycin significantly reduced glucose uptake and glycogen synthesis in cultured rat skeletal muscle cells [20]; pretreatment of HepG2 liver cells with rapamycin resulted in a decrease in glycogen synthase (Gys) activity in an insulinindependent manner [21]. In vivo, rapamycin combined with recombinant human acid $\alpha$-glucosidase (GAA) treatment improved glycogen clearance in the target tissue of adult GAAKO mice; rapamycin alone increased phosphorylation (inactivation) of Gys and reduced glycogen accumulation in skeletal muscles of young GAA-KO mice [22]. Moreover, multiple studies have shown that rapamycin has a strong antifibrogenic effect on liver cirrhosis in rats that underwent bile duct ligation [23-27]. Based on these findings, we predicted that rapamycin could be a potential therapy for GSD III by limiting glycogen synthesis and preventing liver fibrosis. In this study, we investigated whether rapamycin is effective in treating GSD IIIa dogs.

\section{Materials and methods}

\section{Drug preparation}

Rapamycin was purchased from the LC Laboratories (Woburn, MA). For treatment on cultured cells, rapamycin was dissolved in $50 / 50 \mathrm{DMSO} /$ water at $0.5 \mathrm{mg} / \mathrm{ml}$; for use in animals, it was suspended in water at $2-4 \mathrm{mg} / \mathrm{ml}$. All stocks were stored at $-20{ }^{\circ} \mathrm{C}$ until use within 30 days from the preparation date.

Primary culture of skeletal muscle cells from human patients

Myoblasts were isolated from skeletal muscle biopsies of two patients with GSD IIIa and maintained in high-serum growth medium as described, and differentiation of myoblasts into mature myotubes was induced in low-serum differentiation medium [28]. Rapamycin was added to the culture medium at a final concentration of 0.03 or $0.3 \mu \mathrm{M}$. After $48 \mathrm{~h}$, cells were washed three times with cold phosphate buffered saline and then collected with a scraper. Glycogen content was determined in the cell lysates as previously described [28]. A glucose uptake experiment was conducted in live myoblasts as assessed by monitoring accumulation of a fluorescent Dglucose analog, 2-[N-(7-nitrobenz-2-oxa-1, 3-diazol-4-yl) amino]-2-deoxy-D-glucose (2-NBDG, Invitrogen) per manufacturer's instruction, in the presence or absence of rapamycin $(0.03 \mu \mathrm{M})$. Fluorescent cell imaging was obtained using a Nikon DIAPHOT 200 microscope and a Nikon DS-Fil digital camera.

Animals

The canine GSD IIIa breeding colony was maintained at Michigan State University under protocols approved by the MSU Institutional Animal Care \& Use Committee. Affected dogs of ages ranging from 2 to 6 months were transported to Duke University Medical Center for this study. All dogs were on a regular diet throughout the study [13]. All animal experiments were approved by the Institutional Animal Care \& Use Committee at Duke University and were in accordance with the National Institutes of Health guidelines. Blood was collected monthly for routine laboratory testing. Liver and skeletal muscle biopsies were performed as described [13].

\section{Rapamycin treatment of GSD IIIa dogs}

Rapamycin was orally administered with a dosing syringe to three GSD IIIa dogs at a dosage of $1 \mathrm{mg} / \mathrm{kg} /$ day from age 2 to 16 months (early-treatment group). Two other dogs were treated with a low-dosage rapamycin $(0.5 \mathrm{mg} / \mathrm{kg} /$ day $)$ from 8 to 12 months of age, then with a high-dosage rapamycin $(1 \mathrm{mg} / \mathrm{kg} /$ day) from 12.5 to 16 months of age (late-treatment group). Three untreated dogs served as controls; two were followed from age 2 to 16 months, and the third was followed from 6 to 16 months of age. Liver and muscle biopsies were performed for all dogs at age 12 and 16 months as described [13]. A baseline tissue biopsy was also conducted at age 4 months for the two late-treatment dogs and two untreated dogs.

Tissue glycogen content and histology

Fresh tissue specimens were immediately frozen on dry ice and stored at $-80^{\circ} \mathrm{C}$ until use for Western blot or biochemical analysis for glycogen content. Additional tissue samples were placed into $10 \%$ neutral buffered formalin (NBF) for routine histology (trichrome stain) or placed into $3 \%$ glutaraldehyde for periodic acid-Schiff (PAS)/Richardson's staining for high resolution light microscopy (HRLM) as previously described $[13,29]$. Images from histological slides were digitally 
captured with a Nikon DS-Ril digital camera using NISElements D imaging software in conjunction with a Nikon Eclipse E600 microscope.

\section{Western blot}

Tissues or cultured muscle cells were homogenized in RIPA buffer (50 mM Tris-HCl, pH 8.0, 150 mM NaCl, $1 \%$ NP-40 (Igepal CA-630), $0.5 \%$ sodium deoxycholate, $0.1 \%$ SDS and $0.5 \%$ Triton X-100) supplemented with protease inhibitor cocktail (Sigma) and incubated for $15 \mathrm{~min}$ on ice, then centrifuged at $10,000 \mathrm{~g}$ for $15 \mathrm{~min}$ at $4{ }^{\circ} \mathrm{C}$. The protein concentration in the supernatant was measured by Bradford method. Thirty microgram of total protein per lane was resolved in $7 \%$ Tris-acetate gel (Invitrogen) and transferred onto PVDF membrane (Pall Life Sciences). The membrane was blocked with milk, incubated with primary antibody followed by horseradish peroxidase-conjugated secondary antibody. The immune complex was detected using enhanced chemiluminescence (Amersham). Primary antibodies used GLUT1 (\#ab15309), LC3A/B (\#ab58610), and LAMP2 (\#ab37024) antibodies from Abcam (Cambridge, MA); glycogen synthase (\#3886), phospho-glycogen synthase (\#3891), phospho-p70 S6 kinase (\#9243), phospho-S6 (\#4857), and Bcl2 (\#2870) antibodies from Cell Signaling Technology (Danvers, MA); smooth muscle $\alpha$-actin (\#SAB2500963) and $\beta$-actin-peroxidase conjugate (\#A3854) antibodies from Sigma-Aldrich (St. Louis,
MO); and GAPDH (\#NB300-320) antibody from Novus Biologicals (Littleton, $\mathrm{CO}$ ).

\section{Statistical analysis}

The significance of the difference between two groups was assessed using two-tailed, equal or unequal variance student $t$ test. A $p$ value $<0.05$ was considered to be statistically significant.

\section{Results}

Rapamycin reduced glycogen synthesis in primary human GSD III muscle cells through inhibition of glycogen synthase expression and glucose uptake

Primary human skeletal muscle cultures were used as a cellular model. Myoblasts were isolated from skeletal muscle biopsies of two patients with GSD IIIa, and the differentiation of myoblasts into myotubes was induced in low-serum medium [28]. Rapamycin significantly reduced glycogen levels by 41 and $50 \%$, respectively, in the myotubes derived from two GSD IIIa patients (Fig. 1a). A glucose uptake experiment showed that rapamycin at $30 \mathrm{nM}$ markedly reduced uptake of a fluorescent D-glucose derivative, 2-[N-(7-nitrobenz-2oxa-1, 3-diazol-4-yl) amino]-2-deoxy-D-glucose (2-NBDG) by patient myoblasts (Fig. 1b). Immunoblotting revealed that
Fig. 1 Action of rapamycin on primary human GSD III muscle cells. a Myotubes from two GSD IIIla patients (Pt-1 and Pt-2) were treated with 30-nM rapamycin for $48 \mathrm{~h}$ and glycogen content was significantly reduced (by $41 \%$ for Pt-1 and $50 \%$ for Pt-2) in the cell lysates. Average and standard deviation are shown $(n=4$, $\left.{ }^{*} p<0.001\right)$. b Rapamycin markedly inhibited glucose uptake in patient myoblasts as determined using a fluorescent D-glucose analog, 2-[N-(7-nitrobenz-2-oxa1, 3-diazol-4-yl) amino]-2-deoxyD-glucose (2-NBDG, Invitrogen). c Western blot for patient muscle cells $48 \mathrm{~h}$ after rapamycin treatment. Glut-1 glucose transporter 1, Gys glycogen synthase, $p$-Gys phosphoglycogen synthase, $L C 3$ microtubule-associated protein light chain 3, LAMP2 lysosomeassociated membrane protein $2, p$ $S 6$ phospho-S6 ribosomal protein. $\beta$-Actin serves as a loading control
A
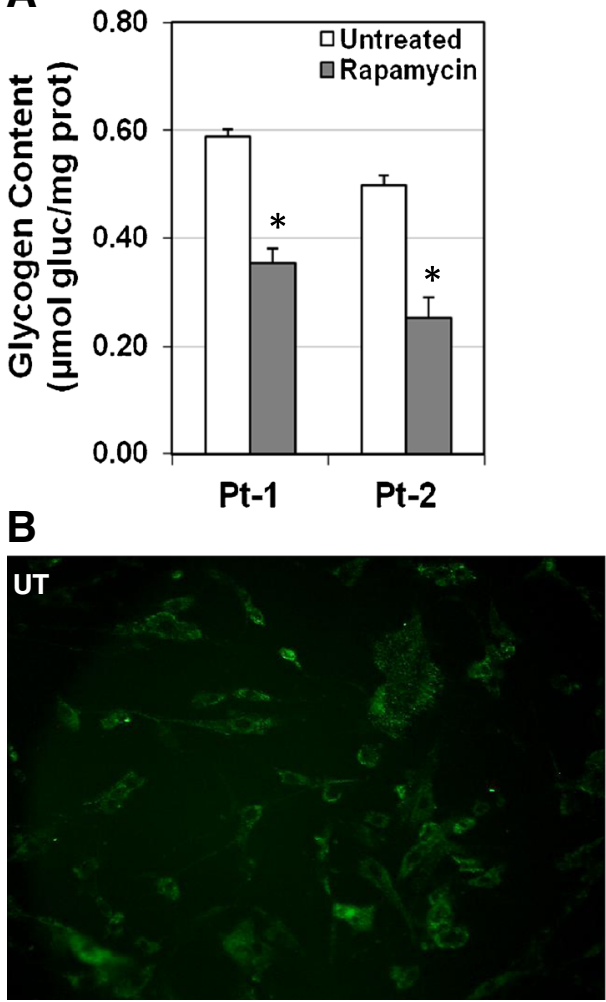
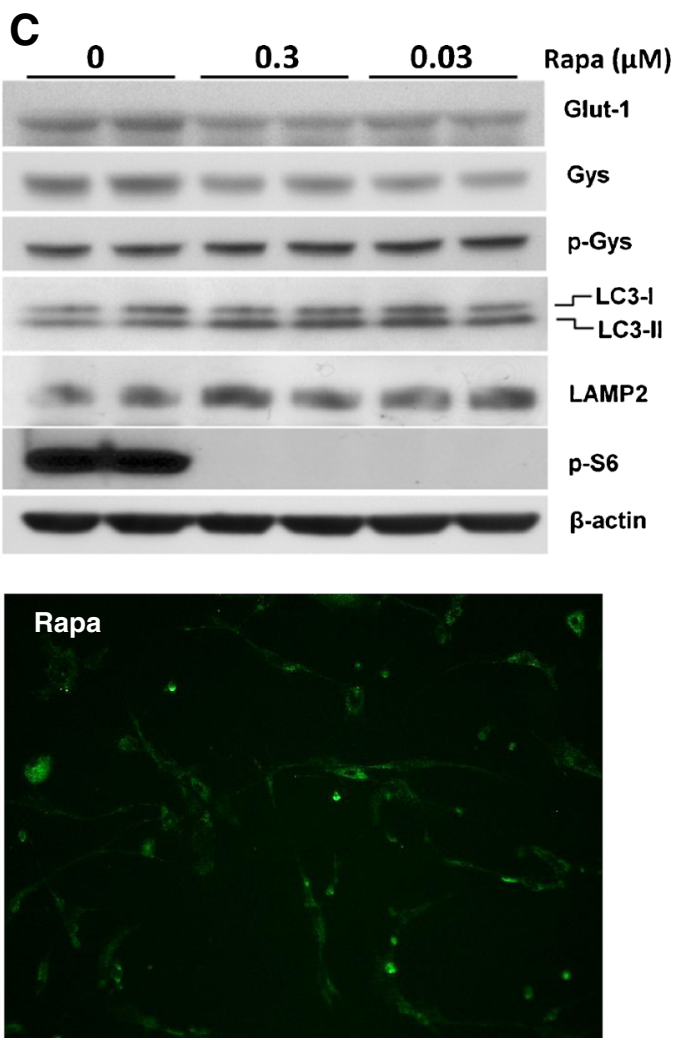
rapamycin decreased glucose transporter Glut-1 and total Gys expression but had no effect on the phosphorylated Gys (pGys, inactive form) level (Fig. 1c). Upregulation of LC3-II (a marker of autophagy) and LAMP2 (a lysosomal marker) in the treated cells suggests that rapamycin treatment could induce autophagy in cultured muscle cells (Fig. 1c). Inhibition of mTOR function by rapamycin treatment was confirmed by the abolished phosphorylation of the ribosomal protein S6, a down-stream target of mTOR (Fig. 1c).

Biochemical correction of glycogen accumulation in muscle and liver of GSD IIIa dogs

To evaluate the efficacy of rapamycin in vivo, three GSD IIIa dogs were administrated rapamycin at $1 \mathrm{mg} / \mathrm{kg} /$ day from age 2 to 16 months (early-treatment group). In untreated dogs, muscle glycogen increased sharply from age 12 to 16 months (Fig. 2a). Rapamycin treatment did not affect muscle glycogen content at age 12 months, but effectively prevented the sharp increase of muscle glycogen content from 12 to 16 months (Fig. 2a). Previously, we demonstrated that liver glycogen content in untreated GSD IIIa dogs peaked at age 12 months but decreased significantly at 16 months due to increased hepatocellular necrosis and subsequent liver fibrosis [13]. Figure $2 b$ shows that liver glycogen was significantly reduced by rapamycin treatment at 12 months (Rapa vs UT). Moreover, there was no obvious change in liver glycogen content between 12 and 16 months in the rapamycin treated dogs in comparison with a sharp drop at 16 months in the untreated dogs.

Next, we decided to determine whether rapamycin could reduce glycogen accumulation in GSD IIIa dogs if treatment was initiated at an older age. Two dogs were treated with rapamycin starting at age 8 months. The initial 4-month treatment with low-dosage rapamycin $(0.5 \mathrm{mg} / \mathrm{kg} / \mathrm{day})$ did not alter the pattern of glycogen accumulation in muscle from age 4 to 12 months (Fig. 2c). Continued treatment with high-dosage rapamycin $(1 \mathrm{mg} / \mathrm{kg} /$ day $)$ reduced muscle glycogen content by 26 and $10 \%$ (16 vs 12 months), respectively, in the two dogs; on the contrary, muscle glycogen increased by 36 and $24 \%$, respectively, in the two untreated dogs (Fig. 2c). Similarly, low-dose treatment had no effect on liver glycogen content at age 12 months; continued treatment with high-dose rapamycin prevented the dramatic reduction of liver glycogen
Fig. 2 Effect of rapamycin treatments on glycogen accumulation in muscle and liver of GSD IIIa dogs. In earlytreatment group ( $\mathbf{a} \& \mathbf{b})$, three affected dogs were treated with rapamycin at $1 \mathrm{mg} / \mathrm{kg} /$ day from 2 to 16 months of age (Rapa). UT untreated group $(n=3), N$ a normal 6-month-old control dog. Tissue biopsies were taken at 12 (12 mo) and 16 months (16 mo) of age. In late-treatment group (c \& d), two dogs (Rapa 1, Rapa 2) were treated with rapamycin at $0.5 \mathrm{mg} / \mathrm{kg} /$ day from 8 to 12 months and at $1 \mathrm{mg} / \mathrm{kg} /$ day from 12.5 to 16 months. UT1 and UT2 are untreated dogs. Tissue biopsies were taken at 4,12 , and 16 months of age for each dog. Glycogen content (micromole glucose released from $1 \mathrm{~g}$ of wet tissue) for each tissue was measured with triplicate specimens. Average plus standard deviation are shown. ${ }^{*} p<0.01$, $* * p<0.05$
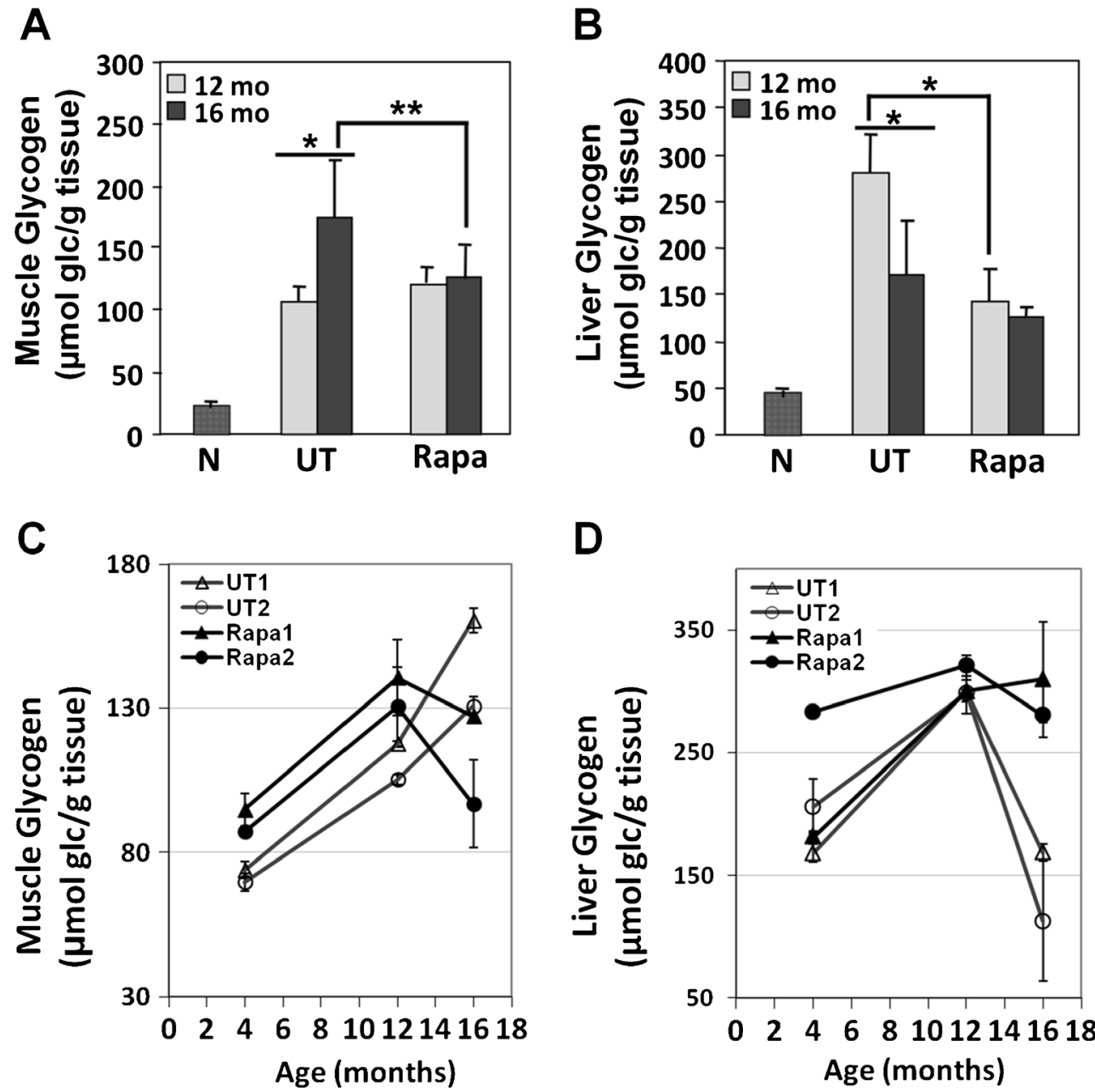

D

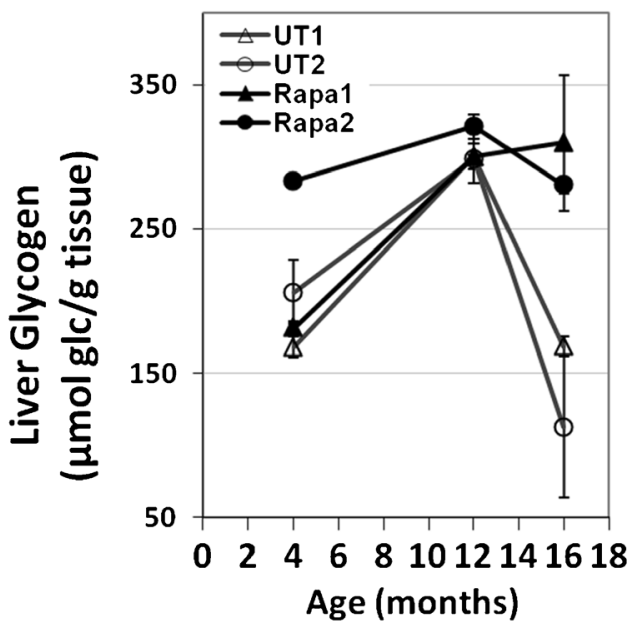


content at 16 months as seen in the untreated dogs (Fig. 2d), indicating that late treatment with rapamycin also prevented replacement of hepatocytes by fibrotic tissue.

Histology confirmed that rapamycin impeded glycogen accumulation in muscle

PAS staining shows more aggressive progression in muscle glycogen accumulation in the untreated $\operatorname{dog}$ (Fig. 3a \& c) than in the rapamycin-treated dog from the early-treatment group (Fig. 3b \& d) from age 12 to 16 months. This pattern was confirmed by the glycogen content analysis in the same dogs (Fig. 3e \& f).

Rapamycin treatment effectively prevented liver fibrosis at advanced age

Trichrome staining of liver sections at 16 months of age showed severely distorted hepatic architecture with dense bands of collagen (bridging fibrosis) in the untreated dog (Fig. 4a \& d), consistent with advanced liver disease (cirrhosis). Rapamycin markedly reduced hepatic fibrosis in both late-treatment (Fig. 4b \& e) and early-treatment (Fig. 4c \& f) groups in which little distortion of hepatic architecture was observed. A better outcome was achieved in the earlier treated dogs.

Consistent with the histological improvement, gross appearance of liver is also greatly improved. As shown in Fig. 5a, an untreated dog (UT) showed enlarged liver with severe, diffuse nodular cirrhosis on surface and in parenchyma at age 16 months; in contrast, rapamycin greatly improved the liver appearance in both early- and late-treatment groups. The rapamycin-treated dogs had larger livers and higher liver:body-weight ratios than those of untreated dogs at age 16 months (Fig. 5b) indicating that less fibrosis was developed in the rapamycin-treated dogs.

In order to study the mechanism of rapamycin action in liver, Western blotting was performed with liver lysates from two untreated and two early treated GSD IIIa dogs, and a normal healthy dog. As shown in Fig. 5c, expression of smooth muscle $\alpha$-actin ( $\alpha$ SMA, a marker of myofibroblasts), Gys, and LC3-II was highly elevated in the liver of untreated
Fig. 3 Representative PAS staining of muscle sections from GSD IIIa dogs. Glycogen staining on muscle biopsies was performed for an untreated dog (a \& c) and an early-treatment dog (b \& d) at age 12 (12 mo) and 16 months $(16 \mathrm{mo})$. Scale bars $=$ $100 \mu \mathrm{m}$. Muscle glycogen content for the same untreated $\operatorname{dog}(\mathbf{e})$ and rapamycin-treated $\operatorname{dog}(\mathbf{f})$ was measured with triplicate tissues. Average plus standard deviation is shown
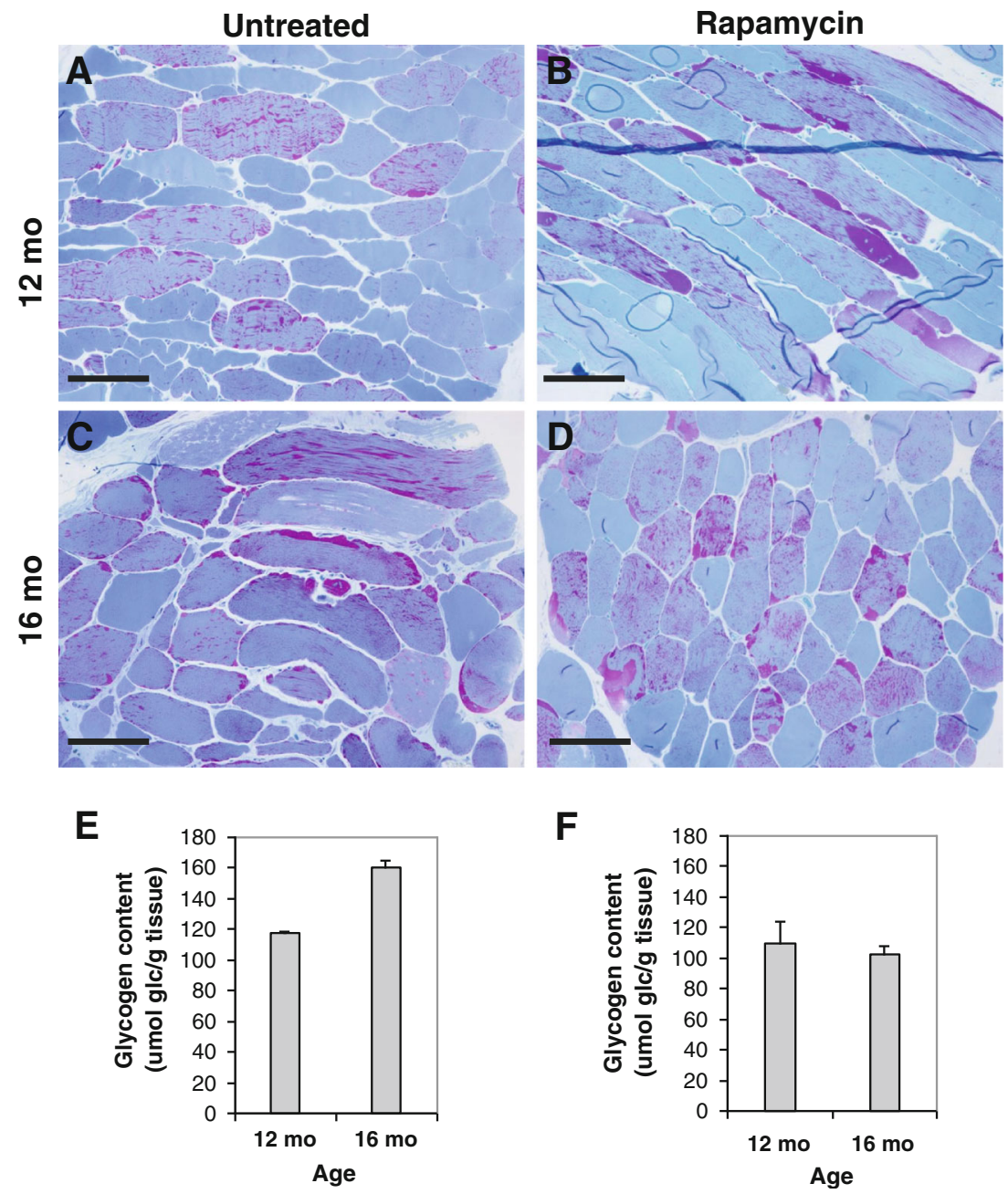
Fig. 4 Representative liver histopathology at 16 months of age. The liver in the untreated $\operatorname{dogs}(\mathbf{a}$ and $\mathbf{d})$ contained dense bands of collagen (bridging fibrosis, blue color), nodule formation, and widespread distortion of the normal hepatic architecture, consistent with advanced liver disease (cirrhosis). The livers of the late-treatment group (b and $\mathbf{e}$ ) and the earlytreatment group (c and $\mathbf{f}$ ) contained areas of fine periportal and pericentral fibrosis, with occasional fine bridging fibrosis. There is little distortion of the normal hepatic architecture in these treated animals. Treatment starting at young age showed a better benefit in the protection of liver (trichrome stain; scale bars in $\mathrm{A}, \mathrm{B}$, and $\mathrm{C}=1,000 \mu \mathrm{m}$; scale bars in $\mathrm{D}, \mathrm{E}$, and $\mathrm{F}=200 \mu \mathrm{m}$ )
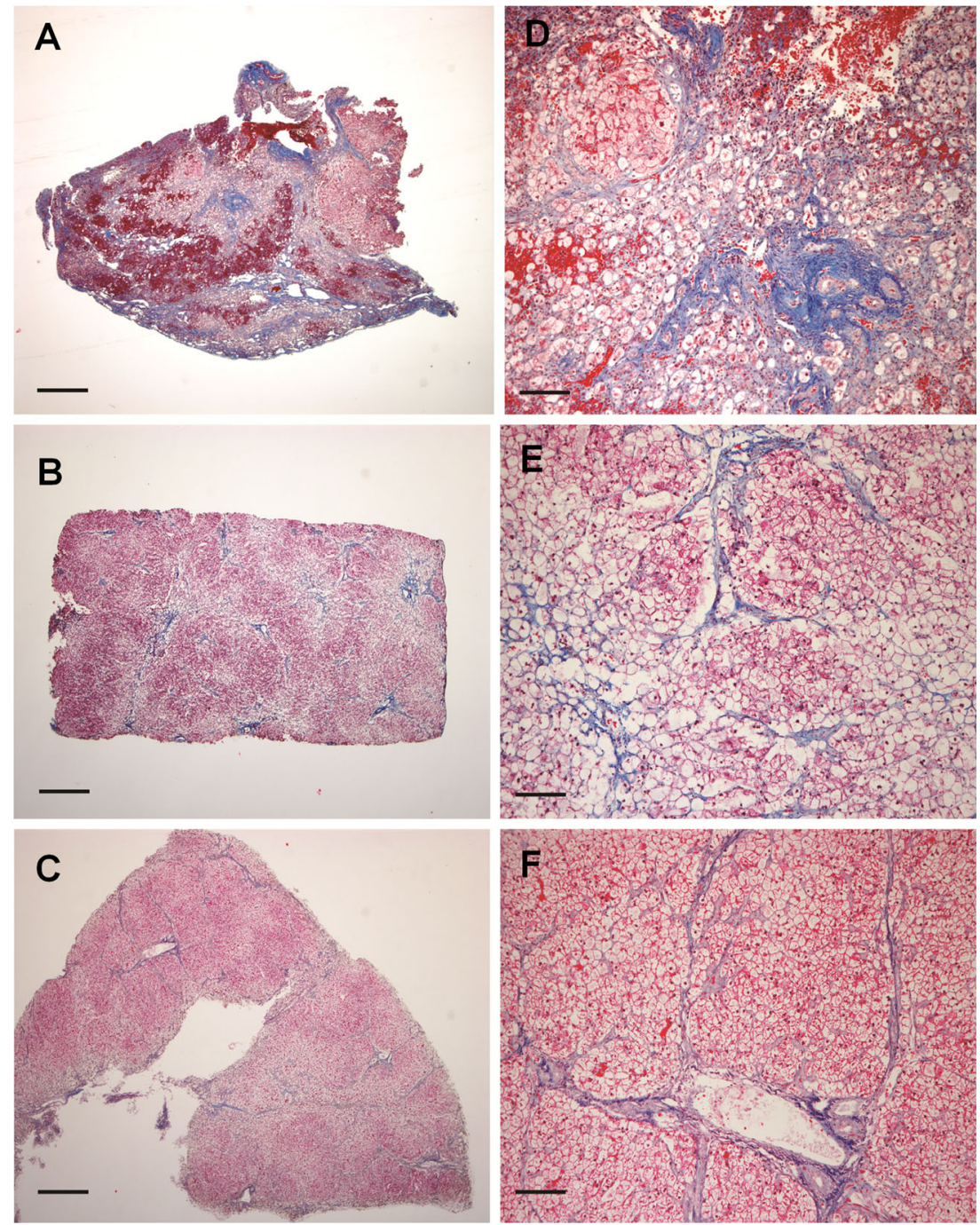

GSD IIIa dogs at age 16 months when compared with the normal healthy dog. Rapamycin treatment markedly lowered the expression of these proteins. The anti-apoptotic protein $\mathrm{Bcl} 2$ was markedly lower in the untreated GSD IIIa dogs than in the normal dog. Rapamycin-treated dogs showed comparable $\mathrm{Bcl} 2$ level to the normal dog. Inhibition of mTOR function was evidenced by decreased phospho-S6 kinase (pS6K), a down-stream target of mTORC1. It seems that mTORC1 activity was strongly induced (increased pS6K levels) in the untreated GSD IIIa dogs in comparison with the normal control dog (Fig. 5c).

\section{Serum biochemistry}

Monthly routine serum biochemistry panels were analyzed for GSD IIIa dogs. For the untreated dogs, liver enzyme activities of AST, ALT, and ALP were elevated above normal range from early ages, and muscle enzyme CPK activity increased above normal range after age 12 month (Fig. 6). Rapamycin treatment had no obvious effect on these enzyme activities.
All other parameters including glucose, triglycerides, cholesterol, bilirubin, albumin, urea nitrogen, and creatinine were within normal ranges for both untreated and rapamycintreated groups (not shown).

\section{Discussion}

Enzyme replacement therapy and gene therapy are useful approaches for treatment of some genetic disorders with single gene deficiency. However, these approaches are not suitable for GSD III due to the lack of receptor-mediated uptake of the enzyme by target cells and the difficulty in the delivery of the large-sized glycogen debranching enzyme expression cassette. Previous studies demonstrated that inhibition of glycogen synthesis is an effective therapeutic approach for Pompe disease [22, 30].

Sipula et al. demonstrated that mTOR inhibition by rapamycin significantly reduced glucose uptake and glycogen synthesis in cultured rat skeletal muscle cells [20]. Ashe et al. 
A

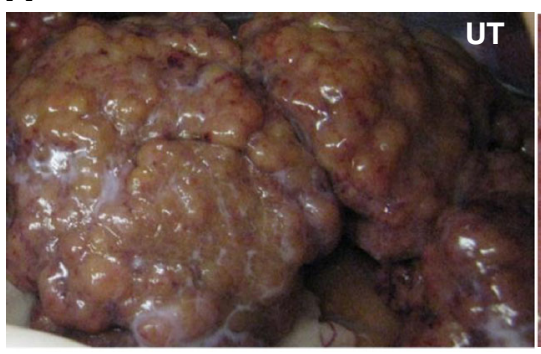

B

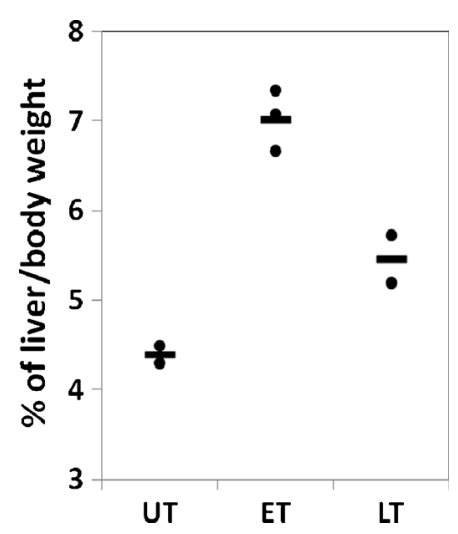

Fig. 5 Rapamycin improved liver symptoms in GSD IIIa dogs at 16 months of age. a An untreated dog (UT) showed enlarged liver with severe, diffuse nodular cirrhosis on the surface; a rapamycin-treated dog (Rapa) from the late-treatment group had a relatively smooth liver surface although some small nodules existed in some regions. b Rapamycintreated dogs showed a higher liver:body-weight ratio when compared with the untreated dogs. Data of each individual dog $(d o t)$ and mean of each group (bar) are shown. UT untreated $(n=3), L T$ late-treatment group

reported that rapamycin reduced lysosomal glycogen accumulation in the skeletal muscle of GAA-KO mice likely through the inhibition of total glycogen synthesis [22]. In this study, we showed that rapamycin significantly reduced glucose uptake and glycogen levels in primary skeletal muscle cells from patients with GSD IIIa, which coincides with the reduction of glucose transporter 1 and total glycogen synthase (Fig. 1). Rapamycin had no effect on the phosphorylation state of Gys in the same experiment (Fig. 1c). In GSD IIIa dogs, rapamycin had little effect on muscle glycogen levels at 12 months of age in both early- and late-treatment groups, but continued treatment prevented the sharp increase of glycogen accumulation from age 12 to 16 months as seen in the untreated dogs (Fig. 2a \& c) [13].

Interestingly, we observed increased expression of LC3-II and LAMP2 in cultured human patient muscle cells (Fig. 1c) and murine $\mathrm{C} 2 \mathrm{C} 12$ cells (not shown) after rapamycin treatment. However, histologically, we did not observe any significant signs of increased autophagy in the muscle of GSD IIIa dogs after rapamycin treatment. Mammucari et al. reported that autophagy in mouse muscle was regulated by mTOR

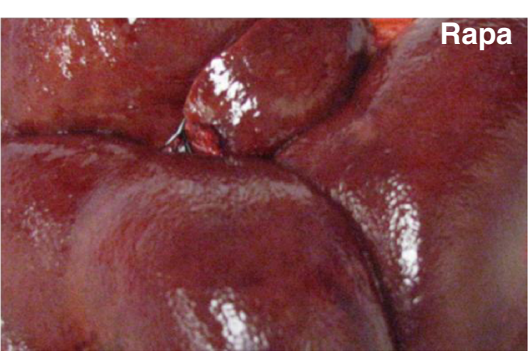

C

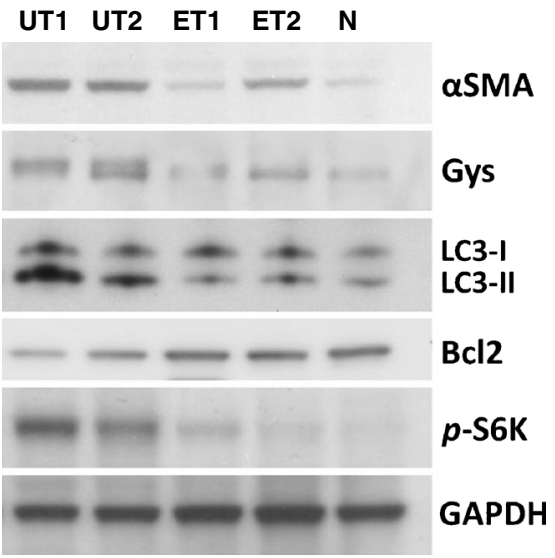

$(n=2), E T$ early-treatment group $(n=3)$. $\mathbf{c}$ Representative Western blot for GSD III dog liver. $\alpha S M A$ smooth muscle $\alpha$-actin, Gys glycogen synthase, $L C 3$ microtubule-associated protein light chain 3, Bcl 2 B-cell lymphoma $2, p-S 6 K$ phospho-S6 ribosomal protein kinase. GAPDH serves as loading control. UT1 and UT2, untreated dogs; ET1 and ET2, rapamycintreated dogs from the early-treatment group; $N$, a normal healthy dog at 6 months of age

complex 2 (mTORC2, rapamycin-insensitive) rather than mTORC1 (rapamycin-sensitive), and injection of rapamycin did not induce autophagy in skeletal muscle in mice [31]. It is well established that mTORC1 directly suppresses autophagy, and mTORC2 may also regulate autophagy via Akt [32]. We did not observe a change in protein levels of p-Akt in human patient muscle cells after rapamycin treatment (data not shown) suggesting that the induction of autophagy in these cells was unlikely regulated by mTORC2-Akt signaling. The discrepancy in rapamycin-induced autophagy between cultured human muscle cells and animal muscle tissue is unclear.

Although hepatomegaly seems improved with age in most patients with GSD III [6], this apparent improvement may be misleading because the decrease in liver size can be a result of progressive liver fibrosis as observed in a GSD IIIa dog model [13]. In untreated GSD IIIa dogs, liver glycogen content peaked at 12 months of age but significantly decreased at 16 months due to large-scale replacement of hepatocytes by fibrous tissue [13]. At age 12 months, liver glycogen content was reduced by $50 \%$ in the early-treatment group (Fig. 2b) but not changed in the late-treatment group (Fig. 2d). Both 
Fig. 6 Analysis of serum enzyme activities in GSD IIIa dogs. Blood was collected every month and routine serum biochemistry determinations were performed. $A L T$ alanine transaminase, $A S T$ aspartate transaminase, $A L P$ alkaline phosphatase, $C P K$ creatine phosphokinase. Values were average \pm standard deviation at each time point in both untreated group (UT, $n=3$ ) and early-treatment group (Rapa, $n=$ 3). Shaded areas indicate normal ranges
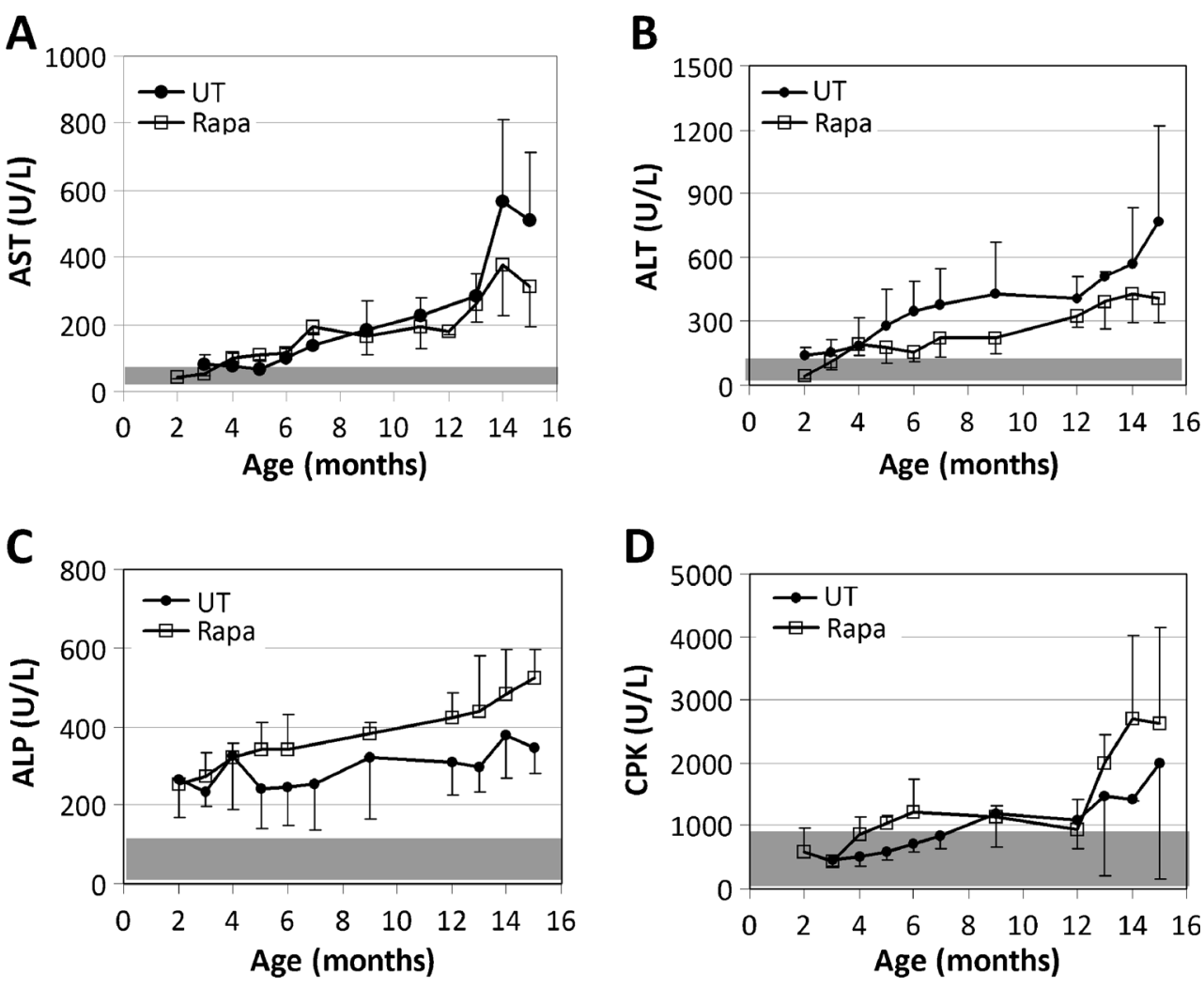

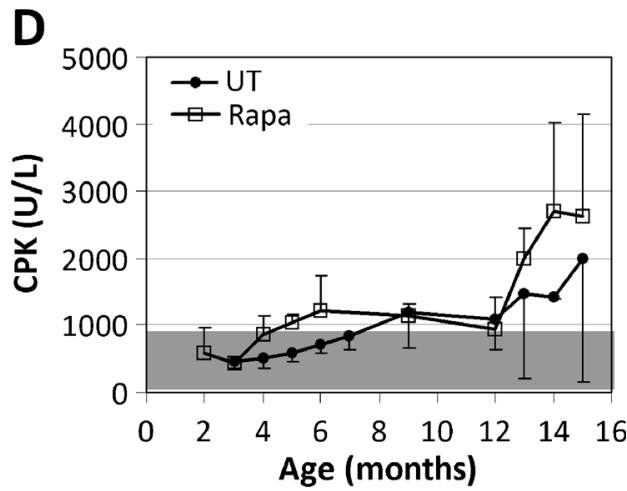

rapamycin treatments markedly reduced liver fibrosis at 16 months (Fig. 4). The rapamycin-treated dogs had larger and softer livers than those of the untreated dogs which could be explained by less fibrosis-induced liver shrinkage. The anti-fibrotic effect of rapamycin was previously reported in several studies using a rat model of liver cirrhosis induced by bile duct ligation [23-27]. To our knowledge, this is the first report of rapamycin effect on liver fibrosis in a large animal model.

Hepatic fibrosis is the consequence of a sustained wound healing response to chronic liver injury from a variety of causes including metabolic diseases like GSD III. Hepatic fibrosis is characterized by accumulation of collagen-rich extracellular matrix, and activation of hepatic stellate cell (HSC) into myofibroblasts is the central event in this process. Myofibroblasts are the major collagen-producing cells in injured liver [33, 34]. In this study, we showed that rapamycin inhibited HSC activation in GSD IIIa dog liver as evidenced by a markedly reduced expression of $\alpha$-SMA (Fig. 5c).

Expression of the autophagy marker LC3-II was highly elevated in the liver of untreated GSD IIIa dogs in comparison with the rapamycin-treated dogs and the normal healthy dog (Fig. 5c). We interpreted that the overload of cytoplasmic glycogen in liver of untreated dogs caused damage to hepatocytes and, subsequently, induced autophagy to sequester the damage. Rapamycin reduced ongoing damage by lowering the glycogen level; therefore, there was less need for the cells to undergo active autophagy to control the damage as evidenced by the reduced level of LC3-II. Interestingly in liver of the untreated dogs, the pS6K levels were greatly higher than that of the normal control dog, indicating induced mTORC1 activity which might be a feedback response to the increased autophagic activities (Fig. 5c). The role of apoptosis in this process is not clear. The anti-apoptotic protein $\mathrm{Bcl} 2$ was markedly reduced in the liver of the untreated dogs, and this reduction was reversed by rapamycin to the similar level of the normal dog (Fig. 5c); however, the apoptosis marker caspase 3 levels were similar in all the dogs (not shown).

The major clinical side-effects of rapamycin are hyperlipidemia and thrombocytopenia $[35,36]$. In this study, serum triglycerides and cholesterol concentrations were within normal ranges throughout the study in both rapamycin-treated and rapamycin-untreated dogs (data not shown). In addition, rapamycin had no obvious effect on growth or serum enzyme activities (Fig. 6).

The major limitation of this study is the small number of animals in each group due to the unaffordable cost for extra dogs, which reduced the significance of important experimental evidence and prevented us to draw a definitive conclusion. A mouse model of GSD IIIa is currently being developed in our laboratory and will be used in the future to confirm our observation.

In summary, rapamycin effectively reduced liver fibrosis and modestly lessened muscle glycogen accumulation in GSD 
IIIa dogs, and our results suggest a potential useful therapy with rapamycin for GSD III. This study, along with previous reports on small animals, strongly indicates that inhibition of mTOR could be an effective treatment approach for liver fibrosis in general. Considering the key role of mTOR in regulating multiple cell functions and the potential side effects of mTOR inhibition, a benefit-risk assessment must be carefully evaluated when translating this therapeutic approach to human diseases.

Acknowledgments We deeply appreciate technical assistance from the Duke DLAR staff including Drs. Feli Smith and Shannon Smith and Ms. Lisa Colvin.

Funding This work was funded by the Alice and Y.T. Chen Research Center for Genetics and Genomics at Duke University and by the Workman family of Lowell, Indiana.

Conflict of interest statement The authors declare no competing or financial interests.

\section{References}

1. Kishnani PS, Austin SL, Arn P, Bali DS, Boney A, Case LE, Chung WK, Desai DM, El-Gharbawy A, Haller R et al (2010) Glycogen storage disease type III diagnosis and management guidelines. Genet Med Off J Am Coll Med Genet 12:446-463

2. Chen YT, Kishnani PS, Koeberl DD (2009) Glycogen storage diseases. In: Valle D, Beaudet A, Vogelstein B, Kinzler K, Antonarakis S, Ballabio A (eds) Scriver's online metabolic \& molecular bases of inherited disease. McGraw-Hill, New York

3. Demo E, Frush D, Gottfried M, Koepke J, Boney A, Bali D, Chen YT, Kishnani PS (2007) Glycogen storage disease type IIIhepatocellular carcinoma a long-term complication? J Hepatol 46: 492-498

4. Cosme A, Montalvo I, Sanchez J, Ojeda E, Torrado J, Zapata E, Bujanda L, Gutierrez A, Arenas I (2005) Type III glycogen storage disease associated with hepatocellular carcinoma. Gastroenterol Hepatol 28:622-625

5. Haagsma EB, Smit GP, Niezen-Koning KE, Gouw AS, Meerman L, Slooff MJ (1997) Type IIIb glycogen storage disease associated with end-stage cirrhosis and hepatocellular carcinoma. The Liver Transplant Group. Hepatology 25:537-540

6. Coleman RA, Winter HS, Wolf B, Chen YT (1992) Glycogen debranching enzyme deficiency: long-term study of serum enzyme activities and clinical features. J Inherit Metab Dis 15:869-881

7. Lucchiari S, Santoro D, Pagliarani S, Comi GP (2007) Clinical, biochemical and genetic features of glycogen debranching enzyme deficiency. Acta myologica: myopathies and cardiomyopathies: official journal of the Mediterranean Society of Myology/edited by the Gaetano Conte Academy for the study of striated muscle diseases 26 : $72-74$

8. Momoi T, Sano H, Yamanaka C, Sasaki H, Mikawa H (1992) Glycogen storage disease type III with muscle involvement: reappraisal of phenotypic variability and prognosis. Am J Med Genet 42 : 696-699

9. Miller CG, Alleyne GA, Brooks SE (1972) Gross cardiac involvement in glycogen storage disease type 3. Br Heart J 34:862-864

10. Olson LJ, Reeder GS, Noller KL, Edwards WD, Howell RR, Michels VV (1984) Cardiac involvement in glycogen storage disease III: morphologic and biochemical characterization with endomyocardial biopsy. Am J Cardiol 53:980-981

11. Moses SW, Wanderman KL, Myroz A, Frydman M (1989) Cardiac involvement in glycogen storage disease type III. Eur J Pediatr 148: 764-766

12. Lee PJ, Deanfield JE, Burch M, Baig K, McKenna WJ, Leonard JV (1997) Comparison of the functional significance of left ventricular hypertrophy in hypertrophic cardiomyopathy and glycogenosis type III. Am J Cardiol 79:834-838

13. Yi H, Thurberg BL, Curtis S, Austin S, Fyfe J, Koeberl DD, Kishnani PS, Sun B (2012) Characterization of a canine model of glycogen storage disease type IIIa. Dis Model Mech 5:804-811. doi:10.1242/ dmm.009712

14. Sengupta S, Peterson TR, Sabatini DM (2010) Regulation of the mTOR complex 1 pathway by nutrients, growth factors, and stress. Mol Cell 40:310-322

15. Rostaing L, Kamar N (2010) mTOR inhibitor/proliferation signal inhibitors: entering or leaving the field? J Nephrol 23:133-142

16. Polak P, Hall MN (2009) mTOR and the control of whole body metabolism. Curr Opin Cell Biol 21:209-218

17. Tsang CK, Qi H, Liu LF, Zheng XF (2007) Targeting mammalian target of rapamycin (mTOR) for health and diseases. Drug Discov Today $12: 112-124$

18. Goberdhan DC, Boyd CA (2009) mTOR: dissecting regulation and mechanism of action to understand human disease. Biochem Soc Trans 37:213-216

19. Dazert E, Hall MN (2011) mTOR signaling in disease. Curr Opin Cell Biol 23:744-755

20. Sipula IJ, Brown NF, Perdomo G (2006) Rapamycin-mediated inhibition of mammalian target of rapamycin in skeletal muscle cells reduces glucose utilization and increases fatty acid oxidation. Metab Clin Exp 55:1637-1644

21. Varma S, Shrivastav A, Changela S, Khandelwal RL (2008) Longterm effects of rapamycin treatment on insulin mediated phosphorylation of Akt/PKB and glycogen synthase activity. Exp Cell Res 314: 1281-1291

22. Ashe KM, Taylor KM, Chu Q, Meyers E, Ellis A, Jingozyan V, Klinger K, Finn PF, Cooper CG, Chuang WL et al (2010) Inhibition of glycogen biosynthesis via mTORC1 suppression as an adjunct therapy for Pompe disease. Mol Genet Metab 100:309-315

23. Patsenker E, Schneider V, Ledermann M, Saegesser H, Dorn C, Hellerbrand C, Stickel F (2011) Potent antifibrotic activity of mTOR inhibitors sirolimus and everolimus but not of cyclosporine A and tacrolimus in experimental liver fibrosis. J Hepatol 55:388398

24. Bridle KR, Popa C, Morgan ML, Sobbe AL, Clouston AD, Fletcher LM, Crawford DH (2009) Rapamycin inhibits hepatic fibrosis in rats by attenuating multiple profibrogenic pathways. Liver Transplant Off Publ Am Assoc Study Liver Dis Int Liver Transplant Soc 15:13151324

25. Neef M, Ledermann M, Saegesser H, Schneider V, Reichen J (2006) Low-dose oral rapamycin treatment reduces fibrogenesis, improves liver function, and prolongs survival in rats with established liver cirrhosis. J Hepatol 45:786-796

26. Biecker E, De Gottardi A, Neef M, Unternahrer M, Schneider V, Ledermann M, Sagesser H, Shaw S, Reichen J (2005) Long-term treatment of bile duct-ligated rats with rapamycin (sirolimus) significantly attenuates liver fibrosis: analysis of the underlying mechanisms. J Pharmacol Exp Ther 313:952-961

27. Zhu J, Wu J, Frizell E, Liu SL, Bashey R, Rubin R, Norton P, Zern MA (1999) Rapamycin inhibits hepatic stellate cell proliferation in vitro and limits fibrogenesis in an in vivo model of liver fibrosis. Gastroenterology 117:1198-1204

28. Sun B, Fredrickson K, Austin S, Tolun AA, Thurberg BL, Kraus WE, Bali D, Chen YT, Kishnani PS (2013) Alglucosidase alfa enzyme 
replacement therapy as a therapeutic approach for glycogen storage disease type III. Mol Genet Metab 108:145-147

29. Lynch CM, Johnson J, Vaccaro C, Thurberg BL (2005) Highresolution light microscopy (HRLM) and digital analysis of Pompe disease pathology. J Histochem Cytochem Off J Histochem Soc 53: 63-73

30. Douillard-Guilloux G, Raben N, Takikita S, Batista L, Caillaud C, Richard E (2008) Modulation of glycogen synthesis by RNA interference: towards a new therapeutic approach for glycogenosis type II. Hum Mol Genet 17:3876-3886

31. Mammucari C, Milan G, Romanello V, Masiero E, Rudolf R, Del Piccolo P, Burden SJ, Di Lisi R, Sandri C, Zhao J et al (2007) FoxO3 controls autophagy in skeletal muscle in vivo. Cell Metab 6:458-471
32. Sridharan S, Jain K, Basu A (2011) Regulation of autophagy by kinases. Cancers 3:2630-2654

33. Safadi R, Friedman SL (2002) Hepatic fibrosis - role of hepatic stellate cell activation. Med Gen Med 4:27

34. Lee UE, Friedman SL (2011) Mechanisms of hepatic fibrogenesis. Best Pract Res Clin Gastroenterol 25:195-206

35. Sacks SH (1999) Rapamycin on trial. Nephrol Dial Transplant Off Publ Eur Dial Transplant Assoc Eur Renal Assoc 14:2087-2089

36. Morelon E, Mamzer-Bruneel MF, Peraldi MN, Kreis H (2001) Sirolimus: a new promising immunosuppressive drug. Towards a rationale for its use in renal transplantation. Nephrol Dial Transplant Off Publ Eur Dial Transplant Assoc Eur Renal Assoc $16: 18-20$ 